\title{
Aquisições no âmbito do Sistema Único de Saúde no Rio de Janeiro: o caso dos programas de atenção básica
}

\author{
Carla Zaire \\ Rondineli Mendes da Silva \\ Lia Hasenclever ${ }^{1}$
}

\begin{abstract}
RESUMO
O objetivo do artigo é comparar os valores de aquisição das compras de medicamentos dos programas de hipertensão, diabetes e asma e rinite do Estado e do Município do Rio de Janeiro com aquelas da Federação. A captura de dados foi realizada nas Secretarias de Saúde do Estado e do Município. Os resultados registraram que o Estado do Rio de Janeiro compra medicamentos a um preço unitário mais alto do que aqueles praticados pelo município e pelo Banco de Preços em Saúde. O Município do Rio de Janeiro tem realizado as compras de medicamentos por licitações na modalidade concorrência e pregão com registro de preços. Esta forma possibilitou a aquisição de medicamentos a preços inferiores aos outros entes pesquisados. A aquisição de medicamentos para a atenção básica pode ser bastante onerosa quando não existe a programação adequada.
\end{abstract}

Palavras-chave: Assistência Farmacêutica; Preços de Medicamentos; Estado do Rio de Janeiro; Município do Rio de Janeiro

\begin{abstract}
The aim of this article is to compare the acquisition value of purchases for medicines programs hypertension, diabetes and asthma and rhinitis from State and City of Rio de Janeiro with those of the federation. Data was collected in Rio de Janeiro State and Municipal Health Secretariats. According to the research, Rio de Janeiro State purchases drugs of higher unit prices than the municipality and the bank. On the other hand, Rio de Janeiro Municipal administration purchases drugs through prices competition and electronic auction, what allowed them to buy drugs of lower prices. The pharmaceutical care in primary health care can be quite costly when there is no proper schedule.
\end{abstract}

Keywords: Pharmaceutical Care; Drug Price; Rio de Janeiro (State); Rio de Janeiro (Municipality).

\footnotetext{
${ }^{1}$ Carla Zaire: Mestre e doutoranda em Economia pelo Centre d'Economie de l'Université Paris Nord-CEPN/ Université Paris 13. Mestre em Saúde Coletiva pelo Instituto de Medicina Social-IMS/Universidade do Estado do Rio de Janeiro-UERJ. carla_zaire@yahoo.com.br
}

Rondineli Mendes da Silva: Mestre e doutorando em Saúde Coletiva pelo Instituto de Medicina Social-IMS/ Universidade do Estado do Rio de Janeiro-UERJ. Analista de gestão em Saúde da Escola Nacional de Saúde Pública-ENSP/Fundação Osvaldo Cruz-FIOCRUZ. rondinelisilva@ensp.fiocruz.br

Lia Hasenclever: Doutora em Engenharia de Produção pela COPPE/Universidade Federal do Rio de JaneiroUFRJ. Professora Associada do Instituto de Economia-IE/Universidade Federal do Rio de Janeiro-UFRJ. lia@ie.ufrj.br 


\section{Introdução}

A distribuição de medicamentos é composta por relações de compra e venda entre empresas comerciais do atacado (distribuidoras) e do varejo (drogarias e farmácias) e as empresas farmacêuticas (laboratórios). Estas realizam vendas sobretudo às empresas distribuidoras e, em menor escala, diretamente às instituições hospitalares públicas e privadas. As distribuidoras fornecem, por sua vez, às farmácias e drogarias e, ainda, às instituições hospitalares públicas e privadas, mas em menor quantidade. Estima-se que em 2001 as distribuidoras de medicamentos compraram $86,5 \%$ da produção das empresas farmacêuticas, dos quais $75,1 \%$ foram vendidos às farmácias e drogarias. As empresas farmacêuticas têm interesse em vender no atacado devido à expressiva economia que fazem em logística e custos de distribuição. Além disso, os grandes laboratórios costumam tirar férias coletivas, o que passa despercebido para os consumidores, quando o estoque é vendido a atacadistas.

O papel das distribuidoras de medicamentos é bem definido no mercado: elas compram das empresas farmacêuticas ao preço de fábrica, impõem sua margem de lucro e depois revendem ao mercado varejo a um preço remarcado. Algumas distribuidoras, após seu estabelecimento no mercado, abriram pontos de venda direta ao consumidor (farmácias). Essas empresas entram com um diferencial significativo no mercado de varejo - como o distribuidor é próprio, é possível que muitos medicamentos cheguem às prateleiras com preços mais baixos em relação aos concorrentes. Exemplo bem conhecido no Rio de Janeiro é a distribuidora Jamyr Vasconcellos, que possui a rede de Drogarias Pacheco. E, ainda, a distribuidora Predimar, que atua nas regiões Sudeste e Sul com sua rede de franquias Farmais (Hasenclever, 2002).

Algumas empresas farmacêuticas atuam nas duas frentes, vendendo a atacadistas e instituições hospitalares públicas e privadas. Neste último caso, ocorre, sobretudo naqueles itens muito caros e importados. Tais itens nem sempre são encontrados em distribuidoras, devido à pouca procura e, portanto, com o risco de perder a validade e acarretar prejuízo. Outras empresas farmacêuticas ainda ganharam mercado pelo diferencial de vender diretamente à rede hospitalar pública e privada - foi o caso da Cristália e da União Química. A Cristália, por exemplo, é a líder no mercado hospitalar doméstico (Magalhães, 2003). Essas empresas ganham clientes, sobretudo, por vender a preços competitivos, possibilitados também pela ausência de intermediários. 
Analisando o mercado de medicamentos do prisma da oferta de produtos, podemos constatar que o Brasil é dominado por aqueles de marca. São oferecidos, segundo a Associação Brasileira do Comércio Farmacêutico, 15.393 medicamentos, dos quais apenas $3.145(20,4 \%)$ são genéricos (ABCFARMA, 2006). Essa multiplicidade de medicamentos presente no mercado brasileiro é negativa segundo diversos pontos de vista. Ocorre prejuízo da fiscalização, do controle da qualidade e dos preços; confunde os consumidores e também os profissionais de saúde; e ainda dificulta o conhecimento suficiente dos produtos para promover uma utilização segura dos mesmos (Nascimento, 2002).

A política de preços de medicamentos no Brasil inclui todos os medicamentos e se aplica a quem vende - seja setor público, setor privado ou ONGs. A margem entre o preço de fábrica e o preço ao consumidor é regulada, variando de acordo com o imposto incidente e o tipo de medicamento. Os impostos que incidem sobre o preço são PIS/COFINS, com alíquota modal de 9,25\%, e ICMS, que varia, de acordo com o estado, de 17 a $19 \%$ - o Estado do Rio de Janeiro especificamente tem a maior alíquota (19\%) (ANVISA, 2011). Eles representaram $33,9 \%$ do custo dos medicamentos, em 2008. Outros produtos, nem sempre essenciais, têm a carga tributária bem menor. São exemplos: teatro e cinema (30,3\%), livros $(15,5 \%)$ e medicamentos de uso animal (13,1\%) (Britto et al., 2012).

Cabe ressaltar que, para entes públicos, existe o Coeficiente de Adequação de Preços (CAP). Este coeficiente é um desconto mínimo obrigatório a ser aplicado pelas distribuidoras e empresas farmacêuticas ao preço de fábrica a produtos vendidos a entes da administração pública. A aplicação do coeficiente estabelece um preço teto para compras governamentais, a partir do qual se deve iniciar o processo de negociação, denominado como Preço Máximo de Venda ao Governo (ANVISA, 2013).

Sendo assim, o objetivo deste artigo é comparar os valores de aquisição das compras de medicamentos dos programas de hipertensão, diabetes e asma e rinite do Estado e do Município do Rio de Janeiro com aquelas da Federação.

Os medicamentos participantes nesta pesquisa de preços são de uso contínuo, utilizados como estratégia de manter maior controle sobre as doenças crônicas não transmissíveis mediante farmacoterapia. Trata-se de aspecto estratégico para a economia da saúde, uma vez que contribui para reduzir os custos sistêmicos com complicações e internações.

Sabe-se que a hipertensão e o diabetes são doenças relacionadas e que quando associadas podem comprometer seriamente a saúde ou pelo menos aumentar 
consideravelmente os riscos de doenças vasculares. O Ministério da Saúde possui um cadastro nacional para pacientes do Programa HiperDia, disponível online. De acordo com informações do próprio site existia em dezembro/2013 mais de 8 milhões de usuários hipertensos e mais de 500 mil diabéticos. Pacientes com hipertensão e com diabetes simultaneamente chegavam a 2,46 milhões (DATASUS-HIPERDIA, 2013). Outra pesquisa, no entanto, aponta para um número bastante expressivo de brasileiros que sofrem de hipertensão e diabetes. A pesquisa Vigitel - por inquérito telefônico, realizada em 2012, aponta 24,3\% da população brasileira com diagnóstico de hipertensão e 7,4\% com diagnóstico de diabetes (BRASIL, 2013). E mais: boa parte dos brasileiros não se trata no SUS e tem dificuldades de acesso a medicamentos fora ou até mesmo inseridos nesse sistema universal. Isso se traduz na compra de medicamentos feita pelo desembolso direto do próprio paciente e/ou família, como já tem sido apontado nos dados das Pesquisas de Orçamentos Familiares de 2003 e de 2008-2009, que demonstraram o impacto que os medicamentos causam nos orçamentos familiares, principalmente das famílias mais pobres que proporcionalmente comprometem muito mais seu orçamento na aquisição de fármacos, e também sobre aquelas famílias de maior renda, pois produtos farmacêuticos também representavam importância significativa (IBGE, 2010, 2004).

A asma é um problema de saúde pública em países desenvolvidos e em desenvolvimento. Apesar de ocorrer em todas as idades, é mais comum na infância. A rinite alérgica pode ser considerada a doença de maior prevalência entre as doenças respiratórias crônicas e, apesar de não estar entre aquelas de maior gravidade, é um problema global de saúde pública. O Brasil ocupa a oitava posição mundial em prevalência de asma, com estimativas para crianças e adolescentes escolares variando de menos que 10 a mais do que $20 \%$ em diversas cidades estudadas, dependendo da região e da faixa etária consideradas. Em 2007, foi responsável por cerca de 273 mil internações, gerando custo aproximado de R \$98,6 milhões para o Sistema Único de Saúde (SUS). Houve 2.500 óbitos, de acordo com o DataSUS, dos quais aproximadamente um terço ocorreu em unidades de saúde, domicílios ou vias públicas (BRASIL, 2010).

Este artigo está dividido em cinco seções, além desta Introdução. Na primeira sessão apresentam-se as responsabilidades da assistência farmacêutica básica. Na segunda seção, a metodologia do trabalho. Na terceira sessão, os resultados da comparação de preços de aquisição por programas selecionados. Na quarta, a discussão e, finalmente, a conclusão. 


\section{Responsabilidades da Assistência Farmacêutica Básica}

O Sistema Único de Saúde, tal como foi concebido na Constituição Federal de 1988, pretende atender desde os mais necessitados aos mais abastados. Do ponto de vista social, foi um grande avanço para um país em desenvolvimento como o nosso. No entanto, temos visto a presença de problemas crônicos no atendimento integral à saúde, seja pelo tempo de espera demasiado por uma consulta médica, seja pela falta de medicamentos essenciais para dispensação aos usuários.

As políticas de assistência farmacêutica norteiam as ações das principais gerências em saúde, sobretudo no que tange a aquisições, logística de distribuição e dispensação de medicamentos. Sendo assim, aprofundar a questão do provimento de medicamentos essenciais à população se mostra não só como um fator importante, mas também como uma forma de racionalizar custos com procedimentos e agravos pela falta de adesão ao tratamento farmacológico.

Entre os vários aspectos relacionados à gestão farmacêutica, este trabalho irá focar na eficiência da gestão de aquisição dos medicamentos. Essa é influenciada pela legislação vigente, modalidade de compra praticada, mas principalmente pela organização de cada ente comprador. Da mesma forma que a assistência farmacêutica lida com o acesso, a questão dos preços de custo dos medicamentos interfere neste acesso, uma vez que, quanto menores os preços, maior a disponibilidade desses medicamentos, no sentido que o fator econômico não será um entrave para aquisição.

Sobre a aquisição de medicamentos no SUS podemos comentar que, de um lado, encontramos os fornecedores: organizações voltadas para vender, constituídas por profissionais treinados para persuadir, geralmente bem remunerados proporcionalmente por volume de vendas. E, do outro, estão instituições de saúde compradoras, que possuem as atividades menos focadas, pouco definidas e profissionalizadas e, consequentemente, funcionários vulneráveis a serem convencidos pelos representantes das empresas farmacêuticas. Outra observação que podemos fazer, mesmo que óbvia, diz respeito ao processo de compra e venda, que deve ser permeado por compromissos claramente estabelecidos entre comprador e fornecedor. Portanto, cabe aos fornecedores cumprir os prazos e os quantitativos acordados, enquanto ao comprador compete pagar os valores previstos nos prazos fixados. 
No Brasil não existe uma legislação específica que defina os termos sob os quais deve ser realizada a aquisição de medicamentos no âmbito público. São utilizadas duas normas gerais para aquisição de bens, sendo uma lei ( $\left.n^{\circ} 8.666 / 93\right)$ e um decreto $\left(n^{\circ} 3.555 / 2000\right.$ que foi convertido na Lei $\left.\mathrm{n}^{\mathrm{o}} 10.520 / 2002\right)$, e outras três portarias do Ministério da Saúde $\left(\mathrm{n}^{\circ}\right.$ $\left.1.818 / 97 ; n^{\circ} 2.814 / 98 ; n^{\circ} 3.765 / 98\right)$ que se referem especificamente a medicamentos. A Lei $n^{\circ}$ 8.666/93 regulamenta o art. 37, inciso XXI, da Constituição Federal, instituindo as normas para licitações e contratos da administração pública. Esta lei regula todas as licitações e contratos na esfera pública, ou seja, não está limitada à área da saúde e muito menos à área de medicamentos e insumos. A recente lei $\mathrm{n}^{\circ}$ 12.401/2011 atualizou a Lei Orgânica da Saúde (8080) e trata sobre a assistência terapêutica e a incorporação de tecnologia em saúde.

Mediante esse arcabouço legal restrito, referente à questão de os medicamentos somente serem mencionados em portarias do Ministério da Saúde, tramita no Congresso Nacional e na Câmara de Deputados o Projeto de Lei $n^{\circ} 3.536 / 2004$, do deputado Walter Feldman. Este projeto dispõe sobre o atendimento de requisitos específicos, nas licitações para a compra de medicamentos e insumos farmacêuticos. Portanto, já existe um esforço para melhorar as regulamentações nesta área.

\subsection{O Estado do Rio de Janeiro}

A população estimada do Estado do Rio de Janeiro é de 15 milhões de pessoas, distribuídas em 92 municípios (IBGE, 2007a). Segundo a Secretaria Estadual de Saúde (SES), a rede estadual é composta por 62 unidades, espalhadas pelas diversas regiões do estado, composta por hospitais, maternidades, institutos, unidades de pronto atendimento, etc (Rio de Janeiro, 2013). Essas unidades são bastante diversificadas quanto às especialidades.

O Estado do Rio de Janeiro, através da Portaria GM n ${ }^{\circ} 1.438 / 2002$, foi habilitado em Gestão Plena do Sistema Estadual, passando a receber financiamento federal direto, mediante contrapartida. Sendo assim, o estado adquiriu responsabilidades e independência na gestão de diversas ações em saúde. A SES, conforme regimento interno, somente realiza compras através da Secretaria de Estado de Administração e Reestruturação (SARE). Cabe à SES coordenar tecnicamente a aquisição de medicamentos, sejam eles para a rede estadual ou para os municípios que não dispõem de mecanismos e/ou competência de repasse federal.

$\mathrm{O}$ ato legal estadual, em vigor no período da pesquisa, que rege as compras de medicamentos é a Resolução SARE $n^{\circ} 3.019 / 2003$, que dispõe sobre os procedimentos operacionais inerentes às modalidades de licitação denominadas pregão comum e pregão 
eletrônico, a que estão sujeitas a administração direta, as autoridades e as fundações, nos termos dos decretos n. ${ }^{\circ} 31.863$ e 31.864 , ambos de 16 de setembro de 2002. A resolução classifica os medicamentos, drogas e insumos farmacêuticos como bens comuns. Desta forma, o pregão é a forma pela qual, teoricamente, foram adquiridos os medicamentos que aqui serão apresentados. O Estado do Rio de Janeiro não faz o Registro de Preços. Existe, apenas, a ata da sessão do pregão contendo o registro de todos os licitantes participantes, das propostas escritas e verbais apresentadas, na ordem de classificação, da análise da documentação exigida para habilitação e dos recursos interpostos. Não existe nenhum dispositivo legal que permita a revisão dos preços quando ocorrer variações nos preços de mercado.

A SES realiza a compra de medicamentos no nível central, com entrega direcionada ao estoque da Central Geral de Abastecimento (CGA). Esse estoque redistribui o quantitativo referente a cada uma das unidades da rede conforme recebe as solicitações. O processo de movimentação de estoque é feito de forma informatizada, permitindo que a SES mantenha certo controle em nível central.

\subsection{O Município do Rio de Janeiro}

A população estimada do Município do Rio de Janeiro, segundo o IBGE, é de 6 milhões de pessoas (IBGE, 2007b). A Secretaria Municipal de Saúde divulga que possui mais de 300 unidades de saúde de diversos tipos de atendimento: hospitais, unidades de prontoatendimento, centros de saúde, clínica da família etc (CNES, 2012). ${ }^{2}$ Portanto, temos uma rede assistencial grande que demanda por recursos de assistência farmacêutica intensamente, exigindo a programação e a gerência no nível central imprescindíveis para o bom abastecimento da rede.

O Município do Rio de Janeiro está habilitado na modalidade Gestão Plena do Sistema Municipal (GPSM), ou seja, possui independência e financiamento direto para realizar as ações de saúde. Diferentemente do Estado do Rio de Janeiro, no município foi consolidado de modo definitivo o Sistema de Registro de Preços para aquisição de bens em geral, medicamentos e outros serviços. Este sistema identifica as possíveis alterações de preços no mercado, possibilitando negociações com os fornecedores registrados na Ata de Registro. No período de 2002-2006, a modalidade de licitação foi a concorrência com posterior assinatura da Ata de Registro de Preços, na qual o fornecedor disponibilizou o preço unitário, o prazo de

\footnotetext{
${ }^{2}$ Cadastro Nacional dos Estabelecimentos de Saúde: http://cnes.datasus.gov.br/Lista_Es_Municipio.asp?VEstado=33\&VCodMunicipio=330455\&NomeEstado=RIO \%20DE\%20JANEIRO (acessado em 23/Jul/2012).
} 
validade da proposta e a quantidade estimada a ser fornecida. A partir de 2007, o pregão foi instituído como modalidade de compra, seguido pelo registro de preço. A Autorização de Fornecimento de Material é feita no nível central, com entrega descentralizada nas unidades assistenciais. Existe atualmente um centro de distribuição, localizado em Jacarepaguá, que recebe e distribui principalmente medicamentos do Componente Básico da Assistência Farmacêutica, para a rede de atenção primária de saúde.

A programação anual de medicamentos e os critérios técnicos dos produtos a serem licitados são estabelecidos pela gerência central de AF (NAF-SMS), entretanto existem instâncias específicas da estrutura de gestão que executam as diversas etapas das compras municipais de medicamentos na Secretaria Municipal de Saúde. Nesse sentido, existe uma gerência de compras, que trabalha de forma informatizada, por meio do sistema de controle, planejamento e distribuição de materiais (SISMED), que emite as ordens de entrega aos fornecedores, os quais, por sua vez, abastecem o almoxarifado farmacêutico e a rede de unidades. Esta ferramenta possibilita verificar a situação das autorizações de fornecimento, quanto a entrega, pagamento ou pendências.

\section{Metodologia da pesquisa}

O objetivo de comparar os valores de aquisição das compras de medicamentos do Estado e do Município do Rio de Janeiro àquelas da Federação exigiu a coleta de dados das compras realizadas de modo centralizado fornecidos pelas próprias Secretarias de Saúde (estadual e municipal). Ainda, a respeito da captação de dados, os mesmos foram coletados independentemente do destino final, ou seja, foram incluídos os consumos hospitalar e ambulatorial de toda a rede assistencial. Foram calculadas as médias ponderadas por medicamento/apresentação por ano pesquisado.

Com o objetivo de comparar essas compras regionais com compras nacionais, foram utilizados também os preços médios unitários anuais das compras realizadas pelo diversos entes federados brasileiros disponíveis no Banco de Preços em Saúde (BPS). Foram calculadas médias anuais (média ponderada) a partir dos registros individuais no BPS, independente da instituição compradora, separados por medicamento/apresentação e ano da aquisição. O BPS disponibiliza dados de licitações em todo Brasil, no website mediante inserção de login e senha, previamente disponibilizados pela gerência. 
No caso do estado, será utilizado o período de 2000 a 2006 e, quanto ao município, de 2002 a 2012. Esta pesquisa foi realizada em duas etapas: como parte de uma pesquisa de mestrado, ${ }^{3}$ contendo dados até 2006; em 2013 estendeu-se a coleta dos dados até 2012. Os períodos serão analisados como um todo, compreendido do dia $1^{\circ}$ de janeiro a 31 de dezembro. A data da compra considerada pela pesquisa é aquela da entrega do item, e não a data de solicitação. Dessa forma, somente contabilizamos o item quando este se encontra em estoque, quando então é solicitada a ordem de pagamento.

A seleção dos itens para a pesquisa seguiu dois critérios: $1^{\circ}$ ) pertencer ao elenco de medicamentos previsto na política de assistência farmacêutica básica; $2^{\circ}$ ) a aquisição do item deve ser de responsabilidade das gerências pesquisadas, através de recursos próprios provenientes do fundo estadual/municipal de saúde, acrescido dos valores do incentivo federal. A seleção contemplou 13 itens distribuídos entre os principais programas de assistência farmacêutica básica do SUS: hipertensão, diabetes, asma e rinite.

Espera-se encontrar, a partir da análise comparativa realizada, as seguintes hipóteses sobre o comportamento dos preços: (a) maior eficiência no processo de compras do município do que o do estado, pelo fato de o primeiro estar mais organizado do que o segundo; (b) a eficiência do gasto pode ser influenciada pela modalidade de compra; (c) os melhores preços são obtidos nas compras de maior volume; (d) a natureza dos fornecedores, se fabricantes ou intermediários comerciais, pode influenciar a modalidade de compra adotada e, portanto, os preços também o são; e (d) se a natureza dos fornecedores influencia no preço dos produtos.

\section{Resultados}

Esta seção apresenta os resultados da pesquisa por programa pesquisado.

\subsection{Programa Hipertensão}

Nesta subseção serão apresentados os resultados referentes aos itens distribuídos para o tratamento da hipertensão, a saber: na classe terapêutica de anti-hipertensivos - metildopa 250mg comprimido, captopril 25mg comprimido, propranolol 40mg comprimido; na classe terapêutica de diurético - hidroclorotiazida 25mg comprimido. A Tabela 1 apresenta os preços unitários dos medicamentos deste programa, para o nível estadual e municipal, e do Banco de Preços em Saúde.

\footnotetext{
${ }^{3}$ Ver tese de mestrado de Zaire (2008).
} 
Os preços apresentados na Tabela 1, de maneira geral, mostram a eficiência das compras municipais pois os preços se parecem bastante aos preços encontrados no BPS. Por outro lado, os preços das compras estaduais são mais altos e chegam a alcançar preços do varejo, como no caso da metildopa em 2005 e 2006 e da hidroclorotiazida em 2006.

Tabela 1. Resumo do Programa Hipertensão

\begin{tabular}{|c|c|c|c|c|c|c|c|c|c|c|c|c|c|c|}
\hline Medicamento & $\begin{array}{c}\text { Preços } \\
\text { Unitários } \\
\text { (R\$) }\end{array}$ & 2000 & 2001 & 2002 & 2003 & 2004 & 2005 & 2006 & 2007 & 2008 & 2009 & 2010 & 2011 & 2012 \\
\hline \multirow{3}{*}{$\begin{array}{l}\text { Metildopa } \\
\text { 250mg }\end{array}$} & BPS & - & 0.083 & 0.099 & 0.103 & 0.089 & 0.078 & 0.069 & 0.0456 & 0.0497 & 0.0564 & 0.0527 & 0.0604 & 0.0769 \\
\hline & Estado & 0.11 & 0.099 & 0.098 & - & 0.158 & 0.28 & 0.28 & - & - & - & - & - & - \\
\hline & Município & - & - & 0.1 & 0.111 & 0.159 & 0.094 & 0.092 & 0.0545 & 0.0518 & 0.0538 & 0.0559 & 0.0559 & 0.0579 \\
\hline \multirow{3}{*}{ Captopril 25mg } & BPS & 0.026 & 0.0216 & 0.019 & 0.023 & 0.01 & 0.014 & 0.014 & 0.0099 & 0.0109 & 0.0114 & 0.01 & 0.0108 & 0.0136 \\
\hline & Estado & 0.023 & 0.0214 & 0.036 & 0.028 & 0.03 & 0.03 & 0.032 & - & - & - & - & - & - \\
\hline & Município & - & - & 0.02 & 0.02 & - & - & - & 0.0103 & 0.0099 & 0.0099 & 0.0107 & 0.0105 & 0.0109 \\
\hline \multirow{3}{*}{$\begin{array}{l}\text { Propranolol } \\
\text { 40mg }\end{array}$} & BPS & 0.013 & 0.0145 & 0.014 & 0.015 & 0.026 & 0.014 & 0.012 & 0.0091 & 0.0098 & 0.0092 & 0.0079 & 0.0103 & 0.0122 \\
\hline & Estado & 0.016 & 0.019 & 0.043 & 0.049 & 0.022 & 0.022 & 0.04 & - & - & - & - & - & - \\
\hline & Município & - & - & 0.01 & - & 0.02 & 0.015 & 0.014 & - & 0.0094 & 0.0088 & 0.008 & 0.0085 & 0.0103 \\
\hline \multirow{3}{*}{$\begin{array}{l}\text { Hidroclorotiazida } \\
25 \mathrm{mg}\end{array}$} & BPS & - & 0.011 & 0.012 & 0.019 & 0.01 & 0.014 & 0.012 & 0.0117 & 0.009 & 0.0088 & 0.0092 & 0.0108 & 0.0117 \\
\hline & Estado & - & 0.04 & 0.044 & 0.036 & 0.036 & - & 0.087 & - & - & - & - & - & - \\
\hline & Município & - & - & 0.018 & 0.018 & 0.018 & 0.02 & 0.019 & 0.0135 & 0.0119 & 0.0097 & 0.008 & 0.0093 & 0.0098 \\
\hline
\end{tabular}

Fonte: Elaboração própria a partir de dados do BPS, Estado e Município.

Com relação às razões entre os preços unitários praticados no município e no estado com o BPS (Tabela 2) podemos identificar alguns pontos de destaque. Para o medicamento metildopa 250mg comprimido, podemos evidenciar que o município comprou a preços semelhantes àqueles apresentados no BPS, apresentando razão próxima de 1 , com exceção dos anos 2004 e 2006. Enquanto o estado chegou a pagar valores muito superiores principalmente nos anos 2004, 2005 e 2006. A princípio, esses aumentos não são justificados, mesmo tratando-se da compra em distribuidoras de medicamentos. Sobre o captopril $25 \mathrm{mg}$ comprimido, podemos ressaltar que o município manteve-se dentro das médias nacionais durante toda a década, inclusive pagando preços inferiores ao BPS (anos 2003, 2009 e 2012). Enquanto o estado pagou mais caro, principalmente no período de 2004 a 2006. O ano 2002 também foi desfavorável mesmo com a compra tendo sido realizada no laboratório oficial da Marinha. 
Tabela 2. Razões dos preços em relação ao BPS

\begin{tabular}{|c|c|c|c|c|c|c|c|c|c|c|c|c|c|c|}
\hline Medicamento & Razões & 2000 & 2001 & 2002 & 2003 & 2004 & 2005 & 2006 & 2007 & 2008 & 2009 & 2010 & 2011 & 2012 \\
\hline \multirow{2}{*}{$\begin{array}{l}\text { Metildopa } \\
\text { 250mg }\end{array}$} & S/Estado & - & 0.8384 & 1.01 & - & 0.563 & 0.278 & 0.246 & & - & & $1-$ & - - & \\
\hline & BPS/Município & - & - & 0.99 & 0.923 & 0.559 & 0.829 & 0.747 & 0.8367 & 0.9595 & 1.0483 & 0.9428 & 1.0805 & 1.3282 \\
\hline \multirow{2}{*}{ Captopril 25mg } & S/LStauo & 1.145 & 1.0093 & 0.542 & 0.813 & 0.343 & 0.467 & 0.432 & - & - & - & - & - & - \\
\hline & BPS/Município & - & . & 0.965 & 1.162 & $=$ & - & - & 0.9612 & 1.101 & 1.1515 & 0.9346 & 1.0286 & 1.2477 \\
\hline \multirow{2}{*}{$\begin{array}{l}\text { Propranolol } \\
40 \mathrm{mg}\end{array}$} & BPS/Estado & 0.78 & 0.7632 & 0.314 & 0.309 & 1.177 & 0.623 & 0.3 & & - & - & - & - & - \\
\hline & BPS/Município & - & - & 1.35 & - & 1.295 & 0.933 & 0.857 & - & 1.0426 & 1.0455 & 0.9875 & 1.2118 & 1.1845 \\
\hline \multirow{2}{*}{$\begin{array}{c}\text { Hidroclorotiazida } \\
25 \mathrm{mg}\end{array}$} & BPS/Estado & {$[-$} & 0.275 & 0.283 & 0.528 & 0.275 & - & 0.137 & $D^{-}$ & - & - & - & - & - \\
\hline & BPS/Município & - & - & 0.687 & 1.061 & 0.566 & 0.675 & 0.632 & 0.8667 & 0.7563 & 0.9072 & 1.15 & 1.1613 & 1.1939 \\
\hline
\end{tabular}

Fonte: Elaboração própria a partir de dados do BPS, Estado e Município.

O medicamento propranolol 40mg comprimido teve preços coerentes na base de dados do município, que chegou mesmo a pagar mais barato que o BPS, enquanto o estado mais uma vez pagou mais caro. No entanto, neste item podemos destacar três anos problemáticos (2002, 2003 e 2006) em que foi pago em torno de 3x o valor do BPS. Para o medicamento hidroclorotiazida $25 \mathrm{mg}$ comprimido, encontramos preços superiores tanto no município quanto no estado, mas enquanto no primeiro a diferença beirava os $50 \%$, no estado aparecia em torno de 3x, com exceção do ano de 2006 que alarmava 8x do preço de comparação nacional.

De maneira geral, podemos observar que o estado compra com frequência em distribuidoras de medicamentos enquanto o município predomina nas empresas farmacêuticas. Ainda é preciso ressaltar que as quantidades compradas pelo estado são consideravelmente inferiores quando comparadas às compras municipais. $\mathrm{O}$ item mais dispendioso para o programa, do ponto de vista do preço unitário, em ambas instâncias de governo, foi a metildopa.

Observamos também que os preços não necessariamente variam de acordo com o tipo de fornecedor, podemos ver distribuidoras praticando preços altos (comparáveis às drogarias) e mesmo preços baixos (inferiores às empresas farmacêuticas). Houve casos em que pedidos de entrega não foram cumpridos tanto por farmacêuticas como por distribuidoras.

Os laboratórios oficiais participaram pouco no abastecimento do programa de hipertensão. O estado comprou destes, tendo sido o laboratório oficial Vital Brazil no ano de 2000 para o item propranolol. Já para o item captopril no ano de 2001 foi o laboratório Farmanguinhos e, em 2002, os laboratórios Farmanguinhos e Marinha. Cabe salientar que os preços unitários praticados por Farmanguinhos e Marinha foram superiores ao BPS. Quanto 
ao município, este comprou, em 2011, hidroclorotiazida do laboratório de Farmanguinhos a um preço inferior ao BPS.

\subsection{Programa Diabetes}

Nesta subseção serão apresentados os resultados referentes aos itens adquiridos para o tratamento de diabetes, como hipoglicemiantes orais: glibenclamida 5mg comprimido e metformina 850mg comprimido, e hipoglicemiante injetável, a insulina humana NPH suspensão injetável $100 \mathrm{UI} / \mathrm{mL}$, em frasco-ampola. A Tabela 3 disponibiliza os preços unitários dos medicamentos hipoglicemiantes, do estado e municipal e Banco de Preços em Saúde.

Para o medicamento glibenclamida 5mg comprimido, os preços apresentados pelo município são bem parecidos com o BPS, com exceção do ano 2007, quando pagou mais caro, e 2011, quando pagou mais barato que o BPS, neste caso a compra foi realizada no laboratório de Farmanguinhos. Ainda na Tabela 3, podemos observar que o medicamento glibenclamida sofre redução no município a partir de 2009.

\section{Tabela 3. Resumo do Programa Diabetes}

\begin{tabular}{|c|c|c|c|c|c|c|c|c|c|c|c|c|c|c|}
\hline Medicamento & $\begin{array}{c}\text { Preços } \\
\text { Unitários } \\
\text { (R\$) }\end{array}$ & 2000 & 2001 & 2002 & 2003 & 2004 & 2005 & 2006 & 2007 & 2008 & 2009 & 2010 & 2011 & 2012 \\
\hline \multirow{3}{*}{$\begin{array}{c}\text { Glibenclamida } \\
5 \mathrm{mg}\end{array}$} & BPS & 0.0123 & 0.0111 & 0.0098 & 0.0131 & 0.013 & 0.0142 & 0.0111 & 0.0068 & 0.0104 & 0.0095 & 0.0078 & 0.009 & 0.0089 \\
\hline & Estado & 0.0264 & 0.0107 & 0.01 & 0.0467 & 0.02 & - & 0.0236 & - & - & - & - & - & - \\
\hline & Município & - & - & 0.0097 & 0.0097 & 0.0098 & - & - & 0.0099 & 0.0096 & 0.0087 & 0.008 & 0.0069 & 0.0077 \\
\hline \multirow{3}{*}{$\begin{array}{l}\text { Metformina } \\
850 \mathrm{mg}\end{array}$} & BPS & - & 0.0889 & 0.0569 & 0.0668 & 0.0578 & 0.0456 & 0.0384 & - & - & - & - & - & - \\
\hline & Estado & - & 0.1208 & 0.147 & 0.0785 & 0.09 & 0.14 & 0.14 & - & - & - & - & - & - \\
\hline & Município & - & - & 0.0739 & 0.0698 & 0.069 & 0.0596 & 0.0498 & 0.0302 & 0.025 & 0.0236 & 0.0224 & 0.0359 & 0.0433 \\
\hline \multirow{3}{*}{$\begin{array}{c}\text { Insulina NPH } \\
100 \mathrm{UI} / \mathrm{mL}\end{array}$} & BPS & - & 13.99 & 14.526 & 16.53 & 21.865 & 17.47 & 9.18 & 21.2 & 6.4397 & 5.6902 & 8.3242 & 9.9822 & 12.019 \\
\hline & Estado & - & - & 27.12 & - & - & - & - & - & - & - & - & - & - \\
\hline & Município & - & - & - & - & - & 24.41 & 24.41 & - & - & - & - & - & 9.3557 \\
\hline
\end{tabular}

Fonte: Elaboração própria a partir de dados do BPS, Estado e Município.

O medicamento metformina 850mg comprimido teve preço bastante irregular para o estado, ocorreram compras emergenciais e até uma aquisição por meio da Prefeitura do Rio de Janeiro (2003), que corresponde a repasse ou doação. A diferença do preço do município em relação ao preço BPS ficou em torno de 30\% superior até 2006 (conforme aponta a Tabela 4). A partir de 2007 não foram encontrados dados no BPS. Por fim, observamos os preços 
praticados para a insulina NPH $100 \mathrm{UI} / \mathrm{mL}$ frasco-ampola e encontramos primeiramente grande irregularidade de aquisições. Isto se explica pelo fato de a insulina ser comprada diretamente pelo MS e, posteriormente, fornecida aos estados e municípios. No caso da insulina NPH ocorreu uma parceria entre a Fiocruz e o laboratório ucraniano Indar para transferência de tecnologia possibilitando aumento na concorrência e uma significativa redução no preço da insulina NPH do mercado brasileiro. Isto pode ser visto nos valores encontrados no BPS a partir de 2008 quando houve uma redução abrupta seguida de uma estabilização num patamar inferior ao inicial.

Tabela 4. Razões dos preços em relação ao BPS

\begin{tabular}{|c|c|c|c|c|c|c|c|c|c|c|c|c|c|c|}
\hline Medicamento & Razões & 2000 & 2001 & 2002 & 2003 & 2004 & 2005 & 2006 & 2007 & 2008 & 2009 & 2010 & 2011 & 2012 \\
\hline \multirow{2}{*}{$\begin{array}{c}\text { Glibenclamida } \\
5 \mathrm{mg}\end{array}$} & BPS/Estado & 0.46591 & 1.03738 & 0.98 & 0.2805 & 0.65 & - & 0.4703 & - & - & - & - & - & - \\
\hline & BPS/Município & - & - & 1.0103 & 1.3505 & 1.3265 & - & - & 0.6869 & 1.0833 & 1.092 & 0.975 & 1.3043 & 1.1558 \\
\hline \multirow{2}{*}{$\begin{array}{l}\text { Metformina } \\
850 \mathrm{mg}\end{array}$} & BPS/Estado & - & 0.73593 & 0.3871 & 0.851 & 0.6422 & 0.3257 & 0.2743 & - & - & - & - & - & - \\
\hline & BPS/Município & - & - & 0.77 & 0.957 & 0.8377 & 0.7651 & 0.7711 & - & - & - & - & - & - \\
\hline \multirow{2}{*}{$\begin{array}{c}\text { Insulina NPH } \\
100 \mathrm{UI} / \mathrm{mL}\end{array}$} & BPS/Estado & - & - & 0.5356 & - & - & - & - & - & - & - & - & - & - \\
\hline & BPS/Município & - & - & - & - & - & 0.7157 & 0.3761 & - & - & - & - & - & 1.2847 \\
\hline
\end{tabular}

Fonte: Elaboração própria a partir de dados do BPS, Estado e Município.

Ainda no caso da insulina, a compra direta nos fabricantes é uma situação pouco identificada para estado e município, visto que a quantidade comprada pode ser muito pequena. Isto ainda dificulta a barganha por preços melhores. A maior compra registrada deste item ocorreu em 2012, pelo município, que pagou preço inferior ao daquele no BPS, diferentemente do que ocorreu nos outros anos em que comprou a preços muitos superiores. Entre 2007 e 2011 não houve compra deste item, provavelmente o Ministério da Saúde repassou esse medicamento. Na realidade, o seu preço fica registrado em ata, para o caso em que haja descontinuidade de repasse do Ministério da Saúde. Sobre o programa de diabetes podemos comentar que o item mais dispendioso, do ponto de vista do preço unitário, em ambas as instâncias de governo foi sem dúvida a insulina NPH suspensão injetável 100 $\mathrm{UI} / \mathrm{mL}$, frasco-ampola. Novamente a participação de laboratórios oficiais foi pequena, apenas o laboratório de Farmanguinhos para o item glibenclamida, no ano de 2001 no estado e no ano de 2011 no município. O preço cobrado por Farmanguinhos, em ambos, foi inferior ao BPS. 


\subsection{Programa Asma e Rinite}

Nesta subseção serão apresentados os resultados referentes aos itens distribuídos para o tratamento de asma e rinite: na classe terapêutica de anti-inflamatório esteróide beclometasona 250mg inalatório de uso oral frasco, beclometasona 50mcg spray inalatório de uso nasal frasco, prednisona $20 \mathrm{mg}$ comprimido e prednisona $5 \mathrm{mg}$ comprimido; na classe terapêutica de broncodilatador - salbutamol 100mcg aerossol inalatório frasco e salbutamol 2mg comprimido. A Tabela 5 traz os preços unitários dos medicamentos dirigidos ao tratamento da asma e rinite, para o nível estadual e municipal e do Banco de Preços em Saúde. Segundo os dados encontrados, as compras do Município e do Estado do Rio de Janeiro desse programa até 2006 foram pequenas e irregulares. Somente a partir de 2007, houve compras regulares pelo município.

\section{Tabela 5. Resumo do Programa Asma e Rinite}

\begin{tabular}{|c|c|c|c|c|c|c|c|c|c|c|c|c|c|c|}
\hline Medicamento & $\begin{array}{l}\text { Preços } \\
\text { Unitários } \\
\text { (R\$) }\end{array}$ & 2000 & 2001 & 2002 & 2003 & 2004 & 2005 & 2006 & 2007 & 2008 & 2009 & 2010 & 2011 & 2012 \\
\hline \multirow{3}{*}{$\begin{array}{l}\text { Beclometasona } \\
\text { 250mcg inalatório } \\
\text { uso oral }\end{array}$} & BPS & - & - & - & - & - & - & - & - & 11.4201 & 19.3644 & 11.9912 & 18.6083 & 23.9486 \\
\hline & Estado & - & - & - & - & - & - & - & - & - & - & - & - & - \\
\hline & Município & - & - & - & - & - & - & - & 24.49 & 16.1283 & 13.8902 & 20.8154 & 20.9 & 25.5789 \\
\hline \multirow{3}{*}{$\begin{array}{c}\text { Beclometasona } \\
\text { 50mcg spray } \\
\text { inalatório uso } \\
\text { nasal }\end{array}$} & BPS & - & - & - & - & - & - & - & - & 20.3707 & 20.125 & 12.3317 & 20.6716 & 22.3821 \\
\hline & Estado & - & - & - & - & - & - & - & - & - & - & - & - & - \\
\hline & Município & - & - & - & - & - & - & - & 23.9 & 21.7031 & 15.6646 & 21.4553 & 17.9 & 18.3147 \\
\hline \multirow{3}{*}{ Prednisona $20 \mathrm{mg}$} & BPS & 0.057 & 0.064 & 0.0597 & 0.0816 & 0.0745 & 0.059 & 0.0486 & 0.0352 & 0.0336 & 0.0357 & 0.0385 & 0.039 & 0.062 \\
\hline & Estado & 0.0747 & 0.0576 & 0.0576 & - & 0.132 & 0.16 & 0.16 & - & - & - & - & - & - \\
\hline & Município & - & - & 0.06 & 0.0723 & 0.12 & 0.086 & 0.065 & 0.037 & 0.0365 & 0.0361 & 0.0385 & 0.0371 & 0.0541 \\
\hline \multirow{3}{*}{ Prednisona 5mg } & BPS & 0.018 & 0.0208 & 0.0199 & 0.0389 & 0.0387 & 0.0249 & 0.0203 & 0.0172 & 0.0157 & 0.0159 & 0.0173 & 0.0169 & 0.0271 \\
\hline & Estado & - & 0.0184 & 0.018 & 0.095 & 0.045 & 0.08 & 0.0993 & - & - & - & - & - & - \\
\hline & Município & - & - & 0.02 & 0.0313 & 0.06 & 0.0388 & 0.0292 & \begin{tabular}{|l|}
0.0174 \\
\end{tabular} & 0.014 & 0.0151 & 0.0168 & 0.017 & 0.018 \\
\hline \multirow{3}{*}{$\begin{array}{c}\text { Salbutamol } \\
\text { aerosol inalatório }\end{array}$} & BPS & - & - & - & - & - & - & 7.69 & 9.743 & 3.6528 & 4.7817 & 3.1445 & 2.5496 & 3.9604 \\
\hline & Estado & - & - & 14.166 & 18.677 & - & - & - & - & - & - & - & - & - \\
\hline & Município & - & - & 7.03 & 9.2949 & 12.24 & 12.2 & 11.27 & 7.5 & 2.8799 & 2.9475 & 2.319 & 3.0853 & 2.98 \\
\hline \multirow{3}{*}{ Salbutamol $2 \mathrm{mg}$} & BPS & 0.0105 & 0.009 & 0.0104 & 0.014 & 0.023 & 0.026 & 0.0147 & 0.0188 & 0.0198 & 0.0163 & 0.0325 & 0.2161 & - \\
\hline & Estado & 0.1 & 0.01 & 0.01 & 0.06 & 0.023 & - & 0.03 & - & - & - & - & - & - \\
\hline & Município & - & - & 0.0099 & 0.0109 & 0.0564 & 0.025 & 0.025 & 0.0162 & - & 0.0188 & - & 0.136 & 0.1439 \\
\hline
\end{tabular}

Fonte: Elaboração própria a partir de dados do BPS, Estado e Município.

Os preços se comportaram de maneira irregular, em alguns anos apresentaram-se mais altos e em outros mais baixos, o que pode ser visualizado no cálculo das razões entre preços dos entes subnacionais com o Banco de Preços em Saúde, como apresentado na Tabela 6. 
Em relação à beclometasona tanto a de $250 \mathrm{mcg}$ quanto a de $50 \mathrm{mcg}$, foram encontrados preços somente no município e no BPS e a partir de 2007/2008. O ano de 2009 foi o único em que os preços do município ficaram inferiores aos do BPS. Os fornecedores foram predominantemente distribuidoras de medicamentos.

\section{Tabela 6. Razões dos preços em relação ao BPS}

\begin{tabular}{|c|c|c|c|c|c|c|c|c|c|c|c|c|c|c|}
\hline Medicamento & Razões & 2000 & 2001 & 2002 & 2003 & 2004 & 2005 & 2006 & 2007 & 2008 & 2009 & 2010 & 2011 & 2012 \\
\hline \multirow{2}{*}{$\begin{array}{c}\text { Beclometasona } \\
\text { 250mcg inalatório uso } \\
\text { oral }\end{array}$} & BPS/Estado & - & - & - & - & - & - & - & - & - & - & - & - & - \\
\hline & BPS/Município & - & - & - & - & - & - & - & - & 0.708 & 1.394 & 0.576 & 0.890 & 0.936 \\
\hline \multirow{2}{*}{$\begin{array}{l}\text { Beclometasona } \\
\text { 50mcg spray } \\
\text { inalatório uso nasal }\end{array}$} & BPS/Estado & - & - & - & - & - & - & - & - & - & - & - & - & - \\
\hline & BPS/Município & - & - & - & - & - & - & - & - & 0.939 & 1.285 & 0.575 & 1.155 & 1.222 \\
\hline \multirow{2}{*}{ Prednisona $20 \mathrm{mg}$} & BPS/Estado & 0.763 & 1.111 & 1.036 & - & 0.564 & 0.369 & 0.304 & - & - & - & - & - & - \\
\hline & BPS/Município & - & - & 0.995 & 1.129 & 0.621 & 0.686 & 0.748 & 0.951 & 0.921 & 0.989 & 1.000 & 1.051 & 1.146 \\
\hline \multirow{2}{*}{ Prednisona $5 \mathrm{mg}$} & BPS/Estado & - & 1.130 & 1.106 & 0.409 & 0.860 & 0.311 & 0.204 & - & - & - & - & - & - \\
\hline & BPS/Município & - & - & 0.995 & 1.243 & 0.645 & 0.642 & 0.695 & 0.989 & 1.121 & 1.053 & 1.030 & 0.994 & 1.506 \\
\hline \multirow{2}{*}{$\begin{array}{c}\text { Salbutamol aerosol } \\
\text { inalatório }\end{array}$} & BPS/Estado & - & - & - & - & - & - & - & - & - & - & - & - & - \\
\hline & BPS/Município & - & - & - & - & - & - & 0.682 & 1.299 & 1.268 & 1.622 & 1.356 & 0.826 & 1.329 \\
\hline \multirow{2}{*}{ Salbutamol 2mg comp } & BPS/Estado & 0.105 & 0.900 & 1.040 & 0.233 & 1.000 & - & 0.49 & - & - & - & - & - & - \\
\hline & BPS/Município & - & - & 1.051 & 1.284 & 0.408 & 1.040 & 0.588 & 1.160 & - & 0.867 & - & 1.589 & - \\
\hline
\end{tabular}

Fonte: Elaboração própria a partir de dados do BPS, Estado e Município.

A prednisona 20mg comprimido apresentou resultados dentro do esperado: o estado tendo preços superiores ao BPS. Destacamos, para o estado, o ano de 2006 quando o preço chegou a ser 4x superior ao BPS. E o município que teve em 2004 a maior diferença de preço em relação ao BPS. Podemos destacar também que, em 2001 e 2002, o estado teve como fornecedor o laboratório oficial de Farmanguinhos, o que possibilitou um preço bastante parecido com o do BPS. A partir de 2007 o município pagou preços inferiores ao BPS.

A prednisona de 5mg comprimido teve preços bastante instáveis no estado: a partir de 2003, houve subidas e descidas abruptas sem fazer qualquer associação com os fornecedores. No período de 2001 e 2002 os preços mantiveram-se próximos aos do BPS, o que poderíamos explicar pelo fornecimento de uma empresa farmacêutica e o laboratório de Farmanguinhos. Em 2003 houve a maior alta registrada: quase 3x sobre o valor do BPS, explicado pelas compras emergenciais em distribuidora e drogaria.

Para o medicamento salbutamol $100 \mathrm{mcg}$ aerossol, os dados também estavam irregulares. Foi possível constatar que o estado, nas únicas compras de 2002 e 2003, comprou o salbutamol aerossol com preços altos, embora não houvesse referência do BPS para 
comparar. O município, por sua vez, consegue preços tão competitivos quanto o BPS, principalmente a partir de 2007. As compras do estado estão ocorrendo predominantemente em distribuidoras e até drogarias, que apresentaram o preço mais caro. Por outro lado, a prefeitura apresentou fornecedores predominantemente diretos (empresas farmacêuticas). A partir de 2008, a prefeitura conseguiu preços inferiores ao BPS, comprando predominantemente nas empresas farmacêuticas.

Para o medicamento salbutamol $2 \mathrm{mg}$ comprimido, o estado aparece com altas expressivas nos anos 2000 e 2003. É interessante observar que, quando o estado teoricamente economizaria mais, ou seja, comprando de um laboratório oficial, no caso, o da Marinha (em 2000), ele pagou mais caro pelo comprimido. Isto contraria as expectativas de preços menores, por ser um laboratório oficial. Em relação aos preços do município, estes se aproximaram consideravelmente dos preços do BPS, com exceção dos anos 2004 e 2006. Em 2011, o município pagou um preço inferior ao BPS.

Podemos ainda chamar a atenção para o município que costuma diversificar seus fornecedores num mesmo ano. Isso é devido ao início e término das licitações, que com frequência tem começado no meio do ano e terminado 12 meses depois, logo no meio do ano seguinte. Outra questão a ser observada é que, diferentemente da maioria dos itens pesquisados neste artigo, o município comprou mais nos fornecedores indiretos, ou seja, em distribuidoras de medicamentos, mas ainda assim conseguiu preços competitivos.

O item mais dispendioso para o programa, do ponto de vista do preço unitário, para o estado, foi o salbutamol $100 \mathrm{mcg}$ aerossol inalatório frasco. Enquanto para o município, o preço unitário mais alto foi do medicamento beclometasona $250 \mathrm{mcg}$ inalatório uso oral. Vale ressaltar ainda que ambos os itens identificados anteriormente utilizam o sistema de liberação de partículas por inalação, ou seja, a conhecida "bombinha" dos asmáticos, portanto, no seu preço já está incluída a tecnologia da liberação das doses.

No Programa Asma e Rinite, chamou atenção o salbutamol 100mcg aerossol inalatório, quanto aos preços médios unitários anuais praticados, que tiveram muita variação: os preços mais caros pagos no período pesquisado foram em 2002 ( $\mathrm{R} \$ 14,166)$ e em 2003 ( $\mathrm{R} \$ 18,677$ ), enquanto que em 2010 foram pagos $\mathrm{R} \$ 2,31$. Com o passar dos anos o preço foi diminuindo, seja por razões de escala, seja pela entrada de outros fabricantes no mercado, que aumentaram a concorrência. A partir de 2007 os preços tornaram-se inferiores aos preços encontrados no BPS. 
Ainda foi possível observar neste programa que o Estado do Rio de Janeiro realizou as compras (entre 2000 e 2006) em 13 distribuidoras de medicamentos, seis empresas farmacêuticas, cinco laboratórios oficiais e três drogarias. A prefeitura realizou as compras equilibradamente em distribuidoras de medicamentos e empresas farmacêuticas, e apenas em um laboratório oficial. Vale registrar que o município realizou predominantemente suas compras a preços inferiores ao estado. Houve ainda itens comprados emergencialmente em drogarias, cujo preço pago, nesse caso, foi próximo ao do mercado de varejo.

Outro ponto importante a ressaltar sobre os laboratórios oficiais é que neste programa houve maior participação (laboratórios de Farmanguinhos e da Marinha), fornecendo principalmente para o estado (2000, 2001 e 2002) e para o município (2002).

\section{Discussão}

Os dados coletados na pesquisa nos permitem constatar que as aquisições têm-se dado numa grande gama de fornecedores, desde empresas farmacêuticas e laboratórios oficiais até distribuidoras de medicamentos e drogarias. Surpreendeu-nos a pequena participação dos laboratórios oficiais, que deveriam suprir prioritariamente a demanda do sistema. Portanto, a Rede Brasileira de Produção Pública de Medicamentos, criada em 2005, que previa a reorganização da produção oficial de medicamentos, ainda não permitiu, mesmo em 2012, observar melhora de suprimento no âmbito do SUS no Estado e no Município do Rio de Janeiro. O mais surpreendente é saber que os laboratórios oficiais brasileiros, em vez de funcionarem como fonte de P\&D e parte do sistema nacional de inovação, como no padrão internacional, surgiram associados ao atendimento à assistência farmacêutica à população e à cobertura das lacunas existentes na produção nacional de vacinas e medicamentos essenciais. Porém este objetivo não está sendo alcançado por boa parte dos laboratórios nacionais, e principalmente por aqueles citados nesta pesquisa.

É interessante observar que o laboratório oficial Vital Brazil vendeu somente o item propranolol ao estado em apenas dois anos: 2000 e 2005. O laboratório Vital Brazil é o laboratório farmacêutico do Estado do Rio de Janeiro, logo este deveria estar produzindo para suprir a demanda estadual. No entanto, houve problemas no repasse de medicamentos, seja por incapacidade de garantir a produção, acarretando a descontinuidade do abastecimento, seja porque o mesmo foi fechado por questões sanitárias (não tinha certificado de boas práticas de fabricação) (Zaire, 2008). 
Em relação ao laboratório oficial Farmanguinhos, este aparece realizando algumas vendas consideráveis ao Estado do Rio de Janeiro, mas poucas ao município. O Laboratório Oficial da Marinha realizou também poucas vendas ao município, em 2002, de salbutamol 2mg comprimido. Já em 2012, foram adquiridos, ainda que em pequena quantidade, o captopril $25 \mathrm{mg}$, metildopa $250 \mathrm{mg}$, prednisona de 5 e $20 \mathrm{mg}$ e propranolol $40 \mathrm{mg}$, todos em comprimidos. A prefeitura justificou que existia um problema de operacionalização da entrega. Assim como os outros laboratórios oficiais, ele não garantia o transporte até cada unidade assistencial. E outra questão inevitável era a questão da competitividade de preços: alguns laboratórios privados apresentavam preços mais baixos que os dos laboratórios oficiais (Zaire, 2008).

Dessa forma, é importante investigar melhor os motivos que fazem os laboratórios oficiais não cumprirem seu papel no fornecimento de medicamentos a preços abaixo dos praticados no mercado atacado e, mesmo do BPS, do qual também participam empresas farmacêuticas e distribuidoras.

Observando as empresas privadas, constatamos que a maioria das vencedoras em licitações está envolvida predominantemente no mercado de venda no atacado; no entanto, não é desprezível a atuação das empresas do varejo, neste caso, as drogarias. É visível a predominância de empresas farmacêuticas nas compras do Município do Rio de Janeiro, enquanto as distribuidoras de medicamentos predominam nas compras do Estado do Rio de Janeiro. Em relação a esta questão, o estado justifica que a maioria dos laboratórios não entram na venda direta, fazendo com que as distribuidoras consigam ser de fato maioria. Outra justificativa se refere à fama de mal pagador que o estado adquiriu ao longo de mais de uma década: existia um perfil diferenciado mais conectado àquelas empresas que corriam o risco (Zaire, 2008).

A presente pesquisa possibilitou confrontar os preços de empresas farmacêuticas e de distribuidoras. O resultado esperado seria a prevalência de preços melhores nos medicamentos fornecidos por empresas farmacêuticas, no entanto, não é verdadeira a ideia de que as distribuidoras necessariamente terão preços superiores aos das farmacêuticas. É possível observar que as distribuidoras de medicamentos praticam preços muito variáveis, dependendo da negociação feita com o fabricante. Houve casos em que a farmacêutica apresentou preço menor que a distribuidora de medicamentos: prednisona 20mg comprimido, prednisona $5 \mathrm{mg}$ comprimido e metformina $850 \mathrm{mg}$. Mas para outro item houve o inverso: as distribuidoras de 
medicamentos apresentaram preços menores que as empresas farmacêuticas, como no caso do medicamento propranolol 40mg comprimido.

De um modo geral, foi possível evidenciar na pesquisa que o Estado do Rio de Janeiro comprou o elenco de medicamentos pesquisados a preços mais caros, quando comparado ao município e ao BPS, no período em análise. Nossa hipótese é de que este preço unitário mais alto se deve às inúmeras compras emergenciais realizadas, que estimularam os fornecedores a compensar a perda de escala. Além disso, como a maioria dos fornecedores foram distribuidoras de medicamentos, elas poderiam estar onerando os preços unitários, pois esses distribuidores tiveram problemas no passado com o cumprimento da dívida pelo estado.

O Município do Rio de Janeiro mostrou-se bastante eficaz no que tange à concorrência de fornecedores; seu sistema de compras tem funcionado de maneira apta a cumprir as exigências inerentes à compra de medicamentos. Logo, o poder público tem conseguido a barganha ao comprar no atacado. Os contratos de mais longo prazo sempre implicam maior capacidade de barganha, pois permitem a otimização dos investimentos pelas empresas farmacêuticas e, nos casos de importação, a inclusão de compensações comerciais (Ávila, 2003).

No que tange às quantidades adquiridas para o Estado do Rio de Janeiro, dois aspectos merecem ser lembrados: o primeiro diz respeito à responsabilidade da compra para os municípios não habilitados na gestão plena, segundo a qual o estado recebe parte do recurso do Ministério da Saúde (Incentivo à Assistência Farmacêutica Básica), compra e distribui aos municípios. Logo, as compras deveriam ser maiores e mais regulares, uma vez que incluem os quantitativos desses municípios.

Em relação ao Município do Rio de Janeiro, a aquisição de medicamentos também se apresentou irregular no que se refere às quantidades. Porém isto não significa que não houve planejamento, pelo contrário, o planejamento permitiu que se comprasse a quantidade que de fato seria utilizada pela rede assistencial.

É interessante destacar que o fato de a pesquisa ter sido realizada em duas partes permitiu o acompanhamento, pelo menos municipal, de uma década de aquisições de medicamentos da atenção básica, de três programas estratégicos para evitar os agravos de doenças crônicas que poderiam levar a internações e complicações para a saúde da população.

Ainda no caso do município, podemos comentar que a partir de 2007 houve uma melhoria no sistema de aquisições, comprovada pelos preços inferiores aos preços encontrados no BPS. Isto pode ser explicado em função do aumento no aporte de recursos na 
assistência farmacêutica básica, com plena descentralização aos municípios na gestão da aquisição de medicamentos, o que propiciou estruturação de programa orçamentário municipal específico para a execução das compras, com total centralização da aquisição e ganhos de escala, ocorridas principalmente a partir de 2006.

Nesse período as licitações de medicamentos ocorreram por meio de pregões presenciais, que, em 2009, passaram a ser totalmente eletrônicos, sempre com registro de preços. E, por fim, como fruto da expansão da rede de atenção primária municipal, decorrente do aumento de cobertura da Estratégia Saúde da Família no Rio de Janeiro, que, até dezembro de 2008, era de 3,5\%, passou-se em 2010 para mais de 30\% de cobertura" (Pinto, 2010).

\section{Conclusão}

Embora esta pesquisa tenha avaliado as compras de medicamentos para três programas de assistência farmacêutica básica, é possível que outros medicamentos estejam sendo comprados de maneira semelhante. Conforme a pesquisa destacou, é grande a variabilidade dos valores gastos a cada ano com cada um dos programas de assistência farmacêutica básica. Inclusive, nem sempre se utiliza todo o orçamento disponível. Isto acontece, pois, se por um lado a aquisição de medicamentos tem funcionado de maneira satisfatória, por outro, o que dita o limite de aquisições é o limite de uso das unidades assistenciais. Porém, não podemos esquecer de mencionar que a cobertura aos pacientes realizada no SUS é muito inferior às estimativas de números de pessoas portadoras das doenças mencionadas nesta pesquisa. Portanto, ainda existe uma demanda não atendida pelo SUS, seja pela falta real de acesso aos serviços e aos medicamentos essenciais e necessários a seu tratamento, seja pela não cobertura de todos as medicações disponíveis no mercado, incluindo aquelas consideradas não essenciais do ponto de vista da saúde pública.

A atenção básica constitui o elo mais barato, ágil e eficaz do sistema, evitando-se a utilização de serviços médicos mais caros e sofisticados. Portanto, melhorias no planejamento ainda são necessárias para possibilitar o financiamento regular dos programas e, consequentemente, viabilizar o fornecimento contínuo necessário de medicamentos (Alves, 2003).

Outra questão que podemos destacar é a de que, no nível de compra governamental, a demanda apresenta baixa elasticidade-preço. Quando a compra é licitada, quem tiver o menor 
preço vence a licitação. Sendo assim, se os fornecedores se articularem para subir os preços, é possível que a compra seja feita, embora o município tenha afirmado que a comissão de licitação realiza o levantamento de preços de medicamentos no mercado a fim de verificar se a empresa está com o preço fora da média (Zaire, 2008).

A estrutura legislativa e de regulação do mercado de medicamentos brasileiro se encontra bastante desenvolvida. No entanto, a má prática da gestão tem comprometido toda a cadeia da assistência farmacêutica. Há uma enormidade de regulamentações específicas, constantemente modificadas e que, muitas vezes, se sobrepõem (Kornis, Braga e Zaire, 2008). Não é trivial entender como se dão as transferências de recursos e muito menos a programação para as licitações. Infelizmente, no setor público brasileiro não existem regras duradouras. As regras mudam a cada mudança de governo, e pior, parece que existe uma aversão a tudo aquilo (normas e procedimentos) herdado da gestão anterior. Com isso, os profissionais são prejudicados pois precisam readaptar-se ao novo ambiente normativo, e as atividades diárias modificadas destroem todo o know-how desenvolvido anteriormente. E, logicamente, na ponta desta cadeia aguarda o paciente com sua prescrição médica, sem qualquer entendimento de por que não será atendido.

As forças de mercado conduzem a indústria farmacêutica e a assistência farmacêutica no SUS a um conflito de interesses. As associações e entidades empresariais pressionam o estado por condições mais favoráveis de produção, seja nos assuntos referentes às importações de insumos e maquinários, seja no registro de produtos e patentes. Enquanto isso, o estado, principal comprador de medicamentos, se vê refém da aquisição de medicamentos, uma vez que sua rede de laboratórios públicos não é capaz de suprir suas necessidades, além de muitas vezes não apresentar preços competitivos ao da indústria. Seja pelo fator de produtividade, seja pela qualidade dos produtos, ou até pela exclusividade de outros (patenteados), a indústria farmacêutica tem demonstrado sua supremacia no mercado farmacêutico brasileiro.

Sendo assim, o Estado brasileiro, através do Ministério da Saúde e da ANVISA, precisa sensibilizar os outros ministérios, como da Fazenda, do Desenvolvimento, Indústria e Comércio Exterior e, ainda, o da Ciência e Tecnologia, para as questões farmacêuticas. Não é possível sustentar um sistema de saúde tal como o nosso, dependendo da importação de insumos e sobretudo das empresas estrangeiras.

Vale considerar que outros trabalhos precisam ser desenvolvidos para dar continuidade a esta temática. É necessário, por exemplo, avaliar se as quantidades licitadas são suficientes. 
E, ainda, se esses medicamentos estão sendo predominantemente absorvidos pela rede hospitalar ou pela rede ambulatorial. Entretanto, as limitações quanto à rastreabilidade, ao controle de estoque nas unidades e à compra descentralizada dificultam a avaliação da dispensação farmacêutica. Muitas unidades controlam seus estoques em fichas de prateleira manuais e arquivo de notas fiscais físicos. Portanto, a intenção de informatizar todas as unidades assistenciais de saúde poderá facilitar essas tarefas de controle de estoque e, consequentemente, aumentará a capacidade de gestão dos seus gerentes.

\section{Referências bibliográficas}

ABCFarma, No 184, Ano 14, Dezembro de 2006.

ALVES, T. N. P. Dispensação de Medicamentos: aspectos da realidade em unidades básicas de saúde de Juiz de Fora - MG. Dissertação (Mestrado em Saúde Coletiva), Instituto de Medicina Social, Universidade do Estado do Rio de Janeiro/UFJF, Juiz de Fora, 2003. $157 \mathrm{p}$.

ANVISA (Agência Nacional De Vigilância Sanitária). Tributos incidentes sobre o setor de produtos para saúde, 2011. Disponível em: $<$ http://portal.anvisa.gov.br/wps/wcm/connect/f0e88a8049c454a8a070a66dcbd9c63c/Mic rosoft+Word++Tributos+Produtos+para+Sa\%C3\%BAde+vs+final.pdf?MOD=AJPERES $>$. Data do Acesso: 23/12/2013.

ANVISA (Agência Nacional De Vigilância Sanitária) 2013. Disponível em: <http://s.anvisa.gov.br/wps/s/r/i6>. Data do Acesso: 15/08/2013.

ÁVILA, J. P. C. Políticas ativas para o desenvolvimento do setor farmacêutico brasileiro: oportunidades e bases conceptuais para a sua formulação. Tese (Doutorado em Saúde Coletiva), Instituto de Medicina Social, Universidade do Estado do Rio de Janeiro, Rio de Janeiro, 2003. 204p.

BRASIL. Ministério da Saúde. Avaliação da Assistência Farmacêutica no Brasil. Brasília: OPAS/MS (Organização Pan-Americana da Saúde/Organização Mundial da Saúde), 2005.

Doenças Respiratórias Crônicas. Cadernos de Atenção Básica n.25. Brasília: Ministério da Saúde, 2010. 
- Vigitel Brasil 2012: vigilância de fatores de risco e proteção para doenças crônicas por inquérito telefônico. Brasília: Ministério da Saúde, 2013.

BRITTO, A.; AMORIM, M.C.S.; PERILLO, E. Tributos e Medicamentos. São Paulo: Interfarma, 2012. ISBN 978-85-7983-264-2.

DATASUS-HIPERDIA, 2013. Disponível em: <http://hiperdia.datasus.gov.br/relproghiperdia.asp> Data de acesso: 20/12/2013.

HASENCLEVER, L. Diagnóstico da Indústria Farmacêutica Brasileira. Brasília/Rio de Janeiro: Unesco/FUJB/IE-UFRJ, 2002. 26p.

IBGE (Instituto Brasileiro de Geografia e Estatística). Pesquisa de Orçamentos Familiares $(P O F)$. Disponível em:

<http://www.ibge.gov.br/home/estatistica/populacao/condicaodevida/pof/2003> Data de Acesso: 05/05/2007.

IBGE (Instituto Brasileiro de Geografia e Estatística).

$-$ as, adolescentes e adultos no

Brasil. Rio de Janeiro: IBGE, 2010.

IBGE (Instituto Brasileiro de Geografia e Estatística). Estados@, 2007a. Disponível em: <http://www.ibge.gov.br/estadosat/> Data do acesso: 25/02/2008.

IBGE (Instituto Brasileiro de Geografia e Estatística). Cidades@ , 2007b. Disponível em: <http://www.ibge.gov.br/cidadesat/default.php> Data do acesso: 25/02/2008.

KORNIS, G.; BRAGA, M. H.; ZAIRE, C. E. F. Os Marcos Legais das Políticas de Medicamentos no Brasil Contemporâneo (1990-2006). Revista de Atenção Primária à Saúde (APS). Vol. 11, n. 1, jan./mar. 2008. ISSN: 1516-7704.

MAGALHÃES, L. C. G. Estratégias Empresariais de Crescimento na Indústria Farmacêutica Brasileira: Investimentos, Fusões e Aquisições, 1988-2002. Ipea, Texto para Discussão n. 995, Brasília, 2003. ISSN 1415-4765.

NASCIMENTO, M. C. A Centralidade do Medicamento na Terapêutica Contemporânea. Tese (Doutorado em Saúde Coletiva), Instituto de Medicina Social, Universidade do Estado do Rio de Janeiro, Rio de Janeiro, 2002. 146p.

PINTO, L. F. Clínicas da família na cidade do Rio de Janeiro: a expansão da atenção primária em saúde em questão. In: ANAIS DO 9O NACIONAL DA REDE UNIDA, 18-21 jul. 
Rio Grande do Sul, Brasil. Porto Alegre: Rede Unidade, 2010. Disponível em:< http://www.redeunida.org.br/congressos-anteriores> Data do acesso: 20/07/2012.

RIO DE JANEIRO (Estado). Secretaria de Estado de Saúde. Unidades da Rede Estadual. 2013. Disponível em: <http://www.saude.rj.gov.br/servicos-assistencia-em-saude.html>. Data do acesso: 12/09/2013.

ZAIRE, C. E.F. A Relação entre a Industria Farmacêutica e a Assistência Farmacêutica no Âmbito do Sistema Unico de Saude (SUS): A compra estadual e minicipal de medicamentos no Rio de Janeiro. Dissertação (Mestrado em Saúde Coletiva), Instituto de Medicina Social, Universidade do Estado do Rio de Janeiro, Rio de Janeiro, 2008. 197p. 